\title{
FRAXE mutation in a mentally retarded subject and in his phenotypically normal twin brother
}

\author{
Cristiana Lo Nigro ${ }^{1}$, Francesca Faravelli ${ }^{1}$, Simona Cavani ${ }^{1}$, Lucia Perroni ${ }^{1}$, Paolo Novello ${ }^{2}$, \\ Mariarosa Vitali ${ }^{3}$, Franca Dagna Bricarelli ${ }^{1}$ and Marina Grasso ${ }^{1}$
}

\author{
${ }^{1}$ Laboratory of Human Genetics, ${ }^{2}$ Departments of Neurology and ${ }^{3}$ Pediatric Neuropsychiatry, Galliera Hospital, \\ Genoa, Italy
}

The FRAXE fragile site, $600 \mathrm{~kb}$ distal to the more common FRAXA, has been reported to be expressed in subjects with mild non-syndromal mental retardation (MR). Amplification of more than $200 \mathrm{GCC}$ repeats, associated with methylation of the adjacent $\mathrm{CpG}$ island at $\mathrm{Xq28}$, leads to the expression of the fragile site. In 1996 a large gene, FMR2, transcribed distally from the CpG island and downregulated by repeat expansion and methylation, was identified. Among 232 mentally retarded patients, tested FRAXA negative, we identified an Italian family segregating a hypermethylated expansion at the FRAXE locus in two dizygotic twin brothers, their sister and their mother. The index case was referred at 23 years of age with severe MR, epilepsy, a dysmorphic face with a high arched palate, marfanoid habitus and hyperreflexia of the lower limbs. His brother was referred to as normal and psychometric tests confirmed he is not mentally retarded. All members of the family underwent FRAXE molecular analysis, after cytogenetic expression of the fraX site and negative FRAXA test. Interestingly, an expansion and a hypermethylation at the FRAXE locus were found in all of them. Fibroblasts from the clinically normal brother were assayed for FMR2 expression and the transcription of the gene was found to be silenced. The presence of a phenotypically normal male with absent FMR2 expression in fibroblasts suggests that the relationship between the FRAXE mutation, FMR2 expression and MR needs to be further investigated. European Journal of Human Genetics (2000) 8, 157-162.

Keywords: FRAXE; mental retardation; genotype/phenotype correlation; genetic counselling

\section{Introduction}

Approximately $3 \%$ of the population suffers from mental impairment; $25-50 \%$ of this is X-linked. ${ }^{1}$

FRAXE is a folate sensitive fragile site in $\mathrm{Xq} 28,600 \mathrm{~Kb}$ distal to the FRAXA. ${ }^{2}$ Molecular characterization revealed that individuals expressing FRAXE had amplification of a GCC repeat adjacent to a CpG island. Normal subjects show 6-25 copies of the repeat, while individuals expressing the fragile site have $>200$ copies and their CpG island is fully methylated. ${ }^{3}$

Several families have been described, in whom expression of the FRAXE fragile site and mental retardation (MR) segregate. ${ }^{4-7}$

Corresponding author: Marina Grasso, Laboratory of Human Genetics, Galliera Hospital, Via Volta 10, 16128 Genova, Italy. Tel: +39105632361; Fax: +39105632628; E-mail: m.grasso@galliera.it

Received 2 October 1999; revised 23 September 1999; accepted 29 September 1999
Recently, a gene associated to FRAXE and designed as FM R2 has been cloned and shown to be expressed in brain and placenta. ${ }^{8,9}$ Loss of FMR2 expression seems to correlate with $(G C C)_{n}$ expansion at FRAXE, suggesting the involvement of this gene in FRAXE-associated mild MR. ${ }^{8-10}$ However, a few phenotypically normal males have been reported with complete methylation at the FRAXE locus and no FMR2 expression. ${ }^{6-8}$

\section{Materials and methods \\ Molecular analyses}

The FRAXE locus was studied with conventional Southern blotting of genomic DNA digested with the restriction enzymes HindIII + Notl and hybridised with the probeOxE.20, as described by Knight et al. ${ }^{3}$ BamHI digestion was performed to confirm the presence of the mutation and to 
exclude the possibility of a rare, previously reported, HindIII polymorphism. ${ }^{11}$

Expression studies by standard RT-PCR were performed on RNA extracted from cultured fibroblast cells using FMR2 specific primer pairs 10-37 and 38-7, as described by Gecz. ${ }^{10}$ As control, connexin26 primersF5 and $\mathrm{R}^{12}$ were used.

\section{Neuropsychological tests}

The phenotypically normal brother was examined by neuropsychological tests to evaluate his general intellectual ability, immediate and short-term memory, visuo-spatial memory, language functions, gnostic and prassic functions.

General intellectual ability was assessed using the Wechsler-Bellevue Adult Intelligence Scale (WB-1). ${ }^{13}$ The memory functions were evaluated using the Wechsler Memory Scale (WMS). ${ }^{14}$ The WMS includes numeric spans, Babcock tales, memory for designs, associations. The visuo-spatial functions were assessed using a visuo-spatial memory test, the Benton Visual Retention Test (VRT). ${ }^{15}$ The language functions were assessed using the Aachen Aphasie Test (AAT). ${ }^{16}$ The test includes items for repetition, denomination of objects, comprehension and spontaneous language. The prassic function was assessed from copying 10 designs. The gnostic function was assessed using the Judgement of Line Orientation (JLO) $)^{17}$ and Facial Recognition (FR). ${ }^{18}$

\section{Results}

The 23-year-old propositus was referred with severe mental retardation (MR) and epilepsy. The parents were unrelated. He had a phenotypically normal dizygotic twin brother and a sister. He had a long face with a high forehead and a prominent jaw, a mild degree of hypertelorism and strabismus, low set ears, hypoplastic alae nasi, a prominent lower lip, a high arched palate and crowded teeth (Figure 1A). His height was $192 \mathrm{~cm}$ and weight $45 \mathrm{~kg}$. He had narrow sloping shoulders, long slender hands and feet with long fingers. Testicular size was normal. On neurological examination, he presented with hyperreflexia of the lower limbs. No instrumental investigation could be performed and IQ could not be evaluated. Differential diagnosis included Lujan-Fryns syndrome and fragile $X$ syndrome.
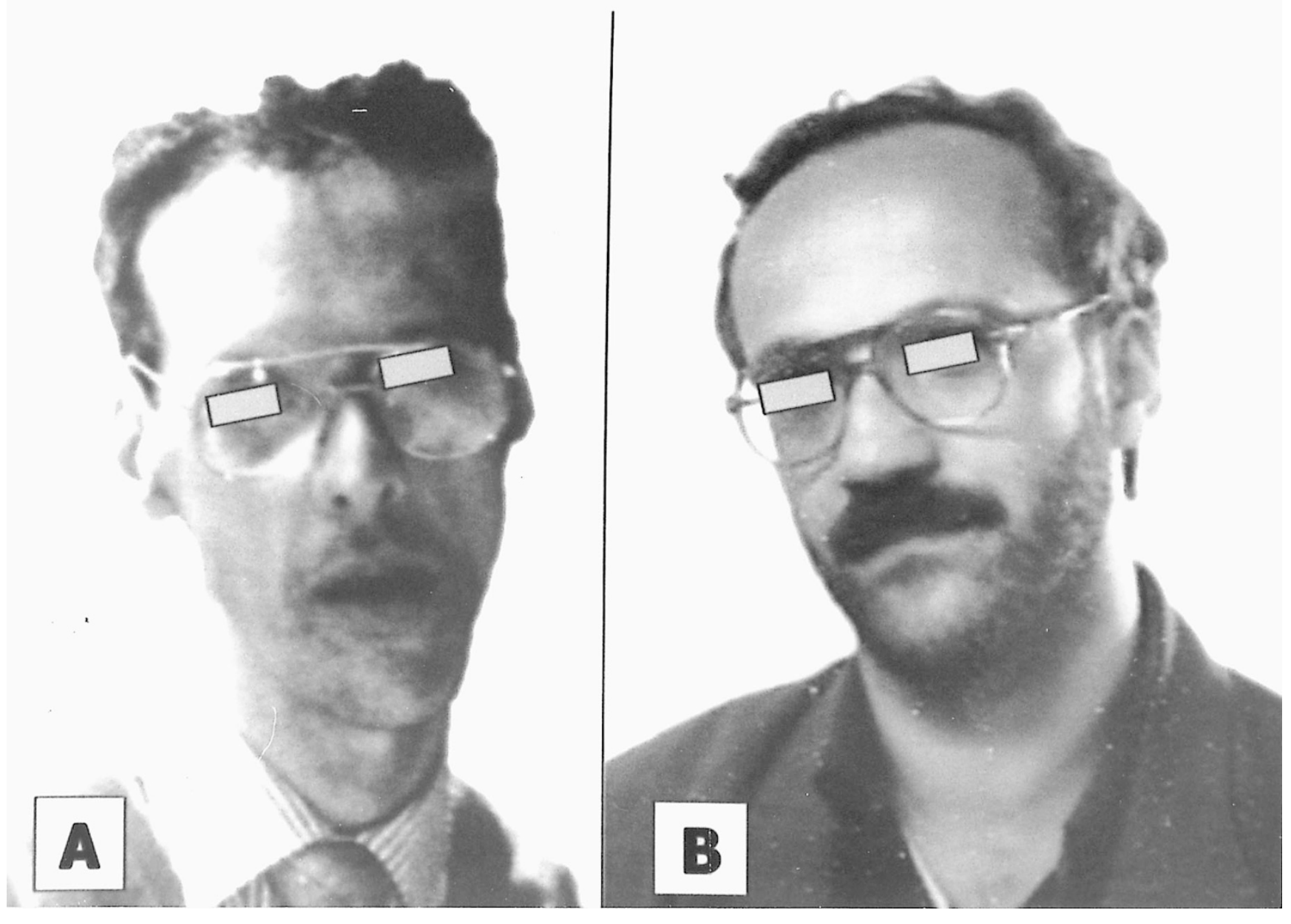

Figure 1 Picture of the proband at the age of 36, A and of the phenotypically normal twin brother at the age of 38, B. 
Cytogenetic analysis revealed a fragile site at Xq27-28 in $18 \%$ of metaphases of the propositus. Similar cytogenetic results were obtained in his phenotypically normal brother, his sister and his mother ( $6 \%, 14 \%$ and $4 \%$, respectively). No expansion was detected in the FMR1 gene (data not shown), thus excluding fragile $X$ syndrome as the cause of mental retardation.

Molecular analysis at the FRAXE locus was therefore performed in the propositus, his siblings and his mother. The results are shown in Figure2. A smear corresponding to a $\Delta>400$ bp demonstrated expansion and methylation in the peripheral blood of the proband (lane4). The hybridisation pattern in the normal brother, sister and mother indicated a

\section{Fam.PGN/84}
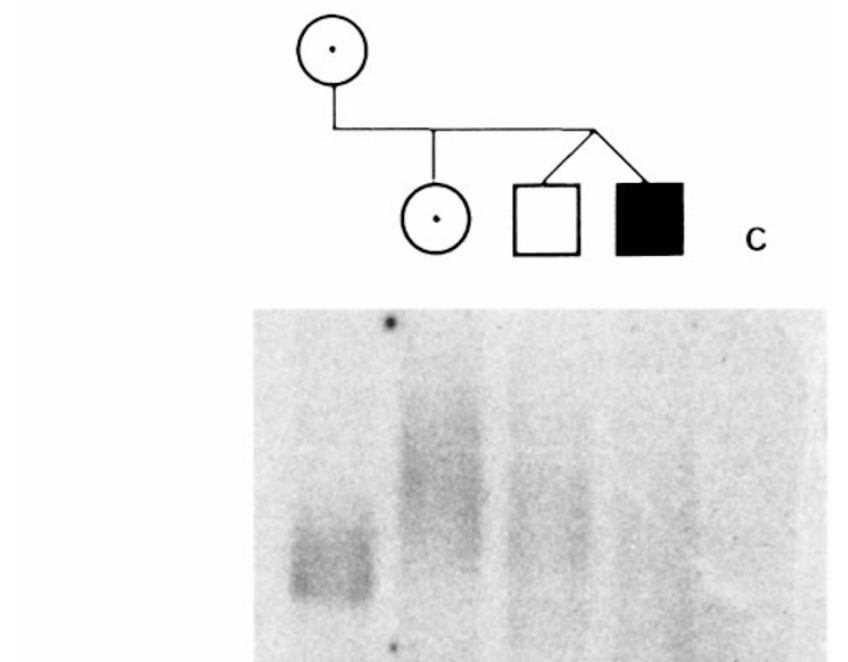

$5.2 \mathrm{~kb} \rightarrow \mathrm{sin}$

$2.8 \mathrm{~kb}$

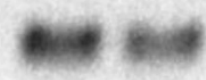

Figure 2 Southern hybridisation of probeOxE.20 to HindIII + Notl digested DNAs from family members is reported below the pedigree. Lane5: control female, in which the $2.8 \mathrm{~kb}$ and the $5.2 \mathrm{~kb}$ bands represent the normal active and inactive allele, respectively. The smears in the other lanes represent the characteristic pattern due to the somatic mosaicism and expansion of the mutated allele in carrier females (lanes 1 and 2) and in FRAXE males (lanes3 and 4). Open circles and square represent normal clinical phenotype. fully methylated genotype, corresponding to a $\Delta>400 \mathrm{bp}$, $\Delta>1000$ bp and $\Delta>800$ bp respectively (lanes $3,2,1$ ). The previously identified HindIII polymorphism at position $14396-97^{11}$ was excluded by Southern blot analysis (data not shown).

A further evaluation of the brother (Figure1B), who is married, has a high school certificate and works as an employee in a school, revealed that none of the clinical features of the proband was present and all the neuropsychological tests performed resulted in the normal range (Table1).

In order to investigate the possibility of a tissutal mosaicism in the normal brother, Southern blot analysis was performed on DNA extracted from fibroblasts and sperm. In fibroblasts a methylated expansion was found ( $\Delta>1500 \mathrm{bp}$ ), whereas in sperm the expansion is lower ( $\Delta=200 \mathrm{bp}$ ) and unmethylated (Figure3).

Cultured skin fibroblast cells from the phenotypically normal brother were analysed by standard RT-PCR (Figure4). Control fibroblasts expressed a high level of FMR2, while the methylated gene of the normal brother showed no expression, as expected since the strong correlation between full methylation and silencing of the transcription of the FMR2 gene in all individuals previously tested. ${ }^{10}$

After genetic counselling, the normal brother and his wife chose to undergo cytogenetic prenatal diagnosis because of advanced maternal age and no FRAXE molecular analysis was performed. Cytogenetic analysis on amniotic fluid revealed a normal female karyotype. At birth FRAXE molecular analysis on cord blood and skin of the daughter revealed a nonmethylated expansion ( $\Delta=200 \mathrm{bp}$ ) of the same size as the father's sperm (Figure5).

Table 1 Results of the neuropsychological tests performed on the phenotypically normal brother

\begin{tabular}{lc}
\hline Overall intelligence (WB-1) & \\
- Verbal I.Q. & 128 \\
- Performance I.Q. & 124 \\
- Total I.Q. & 128 \\
Memory & \\
- Wechsler Memory Scale (WMS) & 80 \\
- Benton Visual Retention Test & 6 \\
$\quad$ (VRT) (no. of errors <16) & \\
Aachen aphasie test (AAT) & No deficit \\
- Spontaneous language & No deficit \\
- Repetition & No deficit \\
- Denomination of objects & No deficit \\
- Comprehension & No deficit \\
- Script language & \\
Prassic function & 13 \\
- Copy of design (n.r. >10) & \\
Gnostic function & \\
- Judgement of line orientation (JLO) (n.r. 25-30) & 28 \\
$\quad$ - Facial recognition (FR) (n.r. 41-54) & 50
\end{tabular}




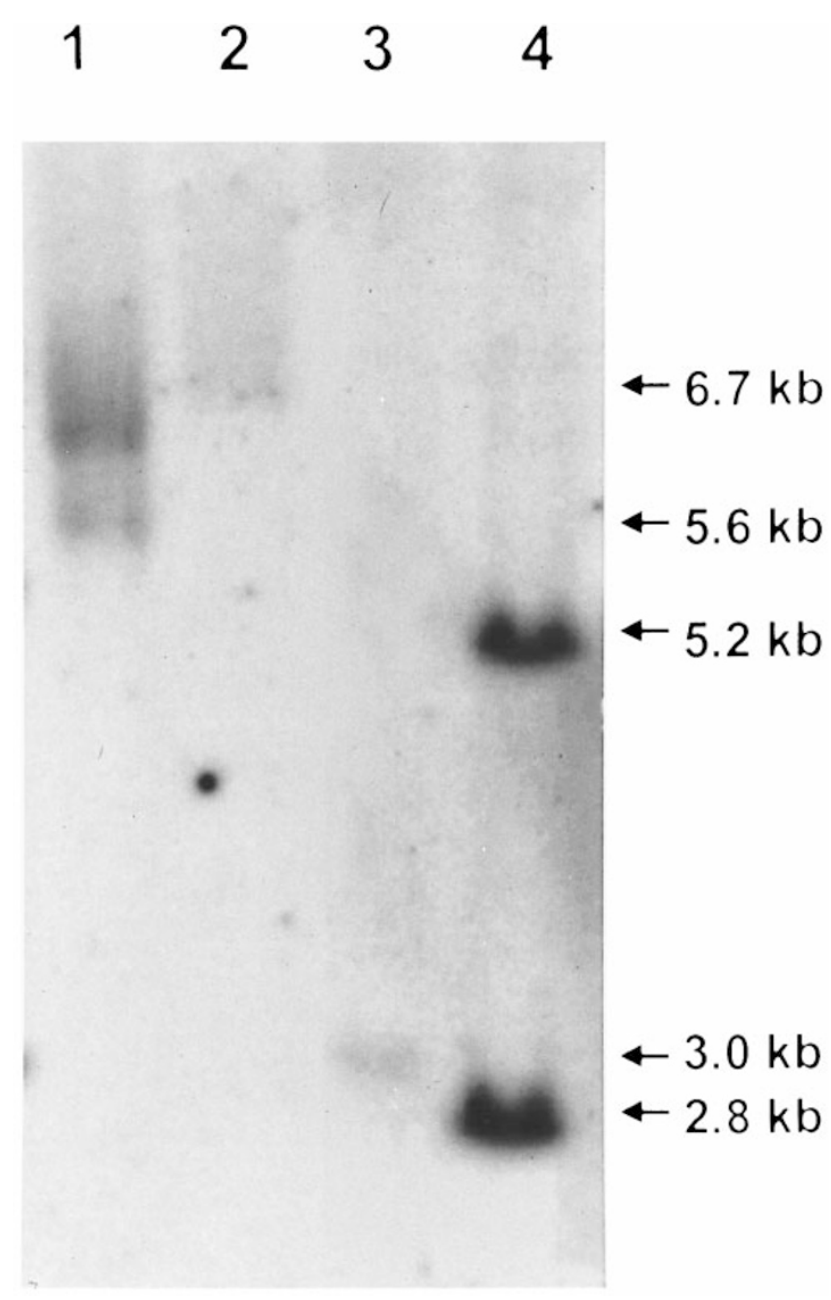

Figure 3 Southern blot analysis on DNAs extracted from whole blood (lane1), skin fibroblasts (lane2) and sperm (lane3) of the phenotypically normal twin brother. Lane4: control female. In lane3, a larger and unmethylated allele was detected.

\section{Discussion}

In the first family reported with FRAXE ${ }^{2}$ the fragile site did not clearly segregate with mental retardation (MR). Further studies on additional families co-segregating $M R$ and the FRAXE fragile site suggested that an etiological relationship may exist between FRAXE and mild non-specific $X$-linked $M R^{4-7}$ The relationship was difficult to establish in the absence of a phenotype other than mild (or borderline) nonspecific MR.

Screening programs in different candidate populations of mentally impaired boys detected $\mathrm{no}^{19-21}$ or only a few cases of FRAXE MR. ${ }^{22-25}$ Based on these studies, the prevalence of FRAXE was estimated to be approximately 1:50000 males. ${ }^{26}$

Here we describe a family segregating a hypermethylated expansion at the FRAXE locus in two dizygotic twin brothers, their sister and their mother. Despite molecular identity, compatible with inactivation of the FMR2 gene and no

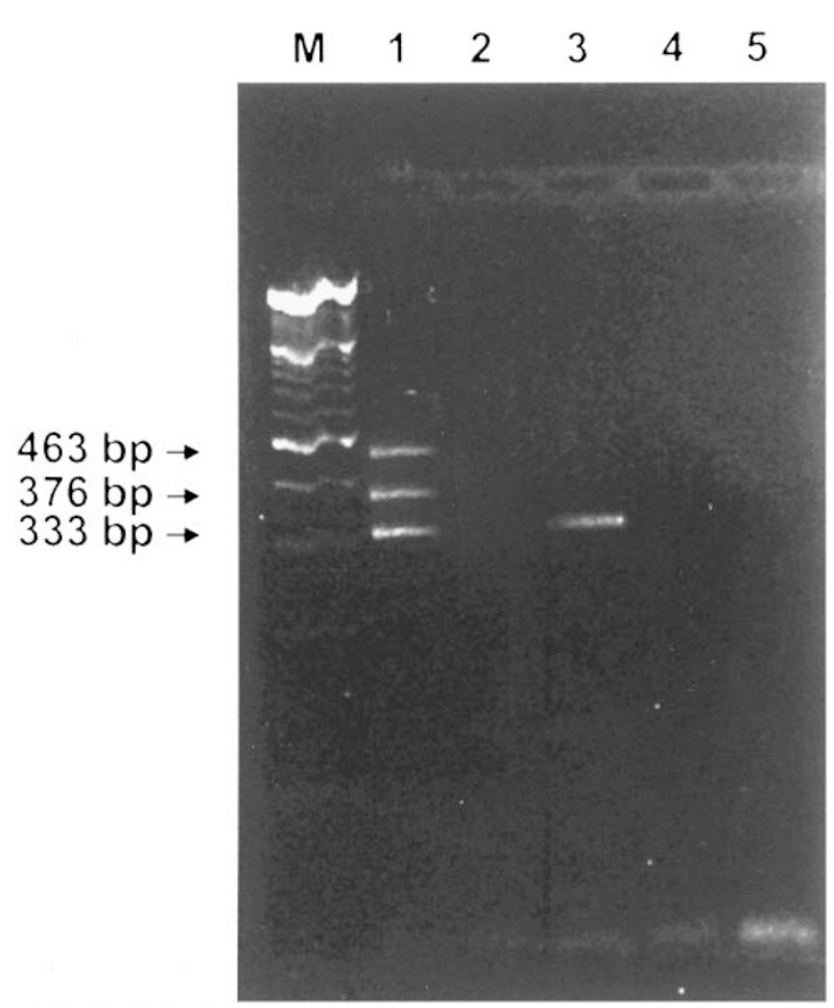

Figure 4 RT-PCR analysis on skin fibroblasts from the phenotypically normal twin brother (lanes 3 and 4) and from a control male (lanes 1 and 2); lanes 1 and 3 are with the presence of reverse transcriptase in the CDNA synthesis reaction $\left(\mathrm{RT}^{+}\right)$, lanes 2 and 4 are the $\mathrm{RT}^{-}$reactions. In lane 5 is the negative control. Marker is the 100 bp ladder.

Amplification products of 463 and $376 \mathrm{bp}$ are obtained from the alternative splicing of FMR2, exon 5 . As control, connexin26 primersF5 and R8 were used (amplicon $333 \mathrm{bp}$ ).

protein production, the two brothers present a very different phenotype: one suffers from severe MR, whereas the phenotype and the intellectual performance of the other appear completely normal.

The hypothesis of a somatic mosaicism of the molecular defect was tested, since specific damage or sparing of a target tissue (especially neuronal) might explain the extreme phenotypic variability observed. The expansion and methylation status were investigated on DNA extracted from fibroblasts and sperm of the clinically normal brother; in fibroblasts a methylathed expansion was revealed ( $\Delta>1500 \mathrm{bp}$ ), whereas an unmethylated fragment of large size ( $\Delta=200 \mathrm{bp}$ ) was observed in sperm. Moreover, expression studies on cultured fibroblast cells from the phenotypically normal brother are consistent with complete inactivation of the FMR2 gene. Other two clinically normal individuals were reported to have the FRAXE CPG island $100 \%$ methylated and, as a consequence, a transcriptionally silent FRM 2 gene. ${ }^{6-8,10}$ Similar cytogenetically positive, fully methylated but unaffected males have been described by Knight $^{4}$ and Murgia; ${ }^{27}$ although FMR2 expression was not 


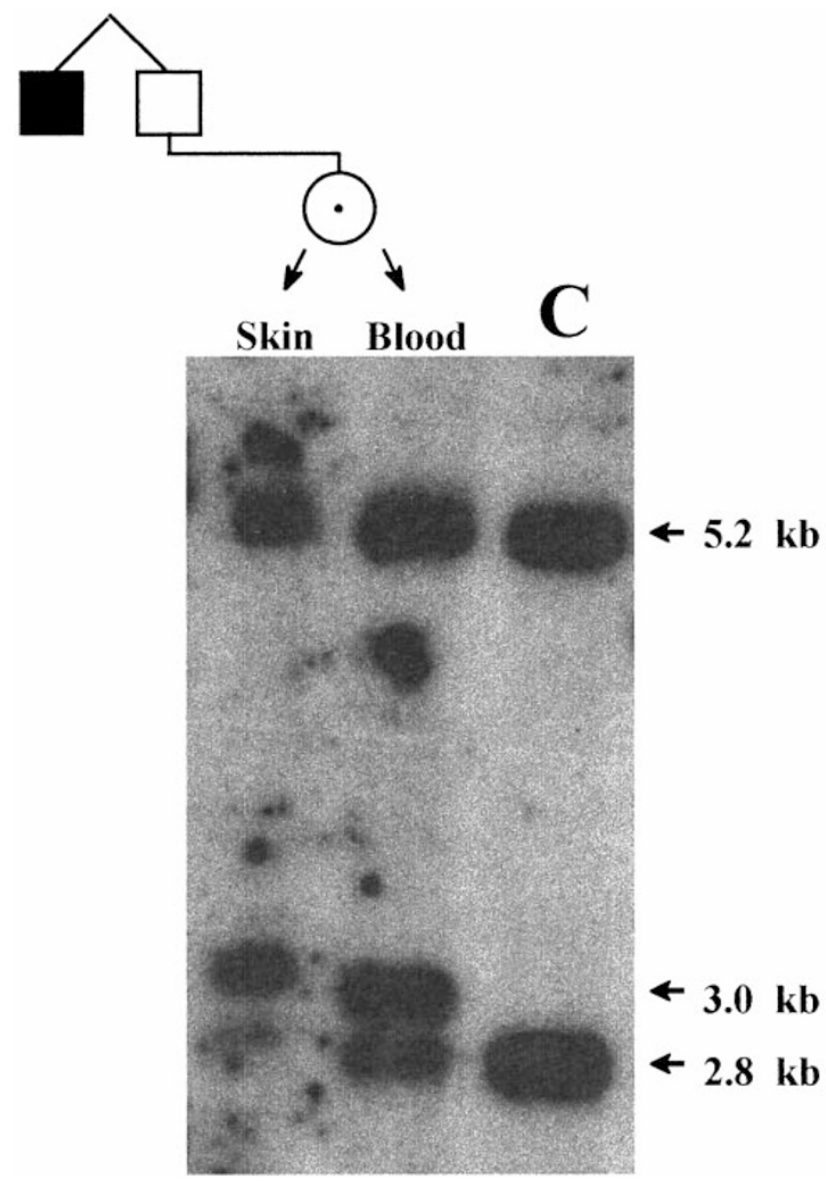

Figure 5 Southern blot analysis on DNAs extracted from cord biopsy (lane 1) and cord blood (lane2) of the clinically unaffected brother's daughter. Lane 3: control female. In lanes 1 and 2 the presence of a larger and unmethylated allele, transmitted through the father's sperm, was revealed. Open square represents normal clinical phenotype.

tested. That could be explained considering that clinical variability may arise if the severity of the FRAXE MR phenotype is compensated by modifying factors present in the genetic background or if the effect of the FRAXE mutation is so mild that the phenotype can remain within the normal range in some subjects. Finally, a genotypephenotype correlation may be confused by the occurrence of mentally retarded subjects unrelated to the fragile site. Considering this, the phenotype of our patient seems to be unusual since mental retardation is severe and dysmorphic features are striking, although Marfan-like habitus, long narrow face, high arched palate and midface hypoplasia have been previously reported in FRAXE patients. ${ }^{28,29}$

Uncertainty about genotype-phenotype correlation in FRAXE-associated MR, resulting from the mild phenotypic effect, the low incidence and the low rate of detection of new cases from large candidate population studies, the variable phenotype of the carrier females and the different size of the expansions transmitted from fully mutated fathers to daughters $^{5-7,26}$ greatly limits the actual possibility of predicting phenotypes in males and females with the mutation and offering prenatal diagnosis to FRAXE families.

In our case, the issue of recurrent risk is raised for the normal brother. The result we obtained on sperm indicates a similarity to FRAXA since males with somatic large methylated expansions are carriers of small unmethylated expansions in germinal cells. ${ }^{30}$ In our family, a small and unmethylated allele was transmitted unmodified from the father to his daughter, at least as far as we can infer from the molecular analysis performed on cord blood and skin.

In conclusion, we think that further studies are needed to evaluate the frequency of the FRAXE mutation both among large samples of mentally retarded subjects and in the general population. Moreover, extensive psychometric testing with expression studies in FRAXE-carrying individuals and in related normal controls would be helpful to evaluate the extent of this mental retardation phenotype.

\section{Acknowledgements}

Weare grateful to the family described here for their kind co-operation. This work was supported by Galliera Genetic Bank (Telethon Project C34). We also thank Dr SJL Knight for the OxE.20 probe.

\section{References}

1 Stevenson R: Mental retardation: overview and historical perspective. Proc Greenwood Genet Ctr 1996; 15: 19-25.

2 Sutherland GR, Baker E: Characterization of a new rare fragile site easily confused with the fragile X. Hum Mol Genet 1992; 1: 111-113.

3 Knight SJL, Flannery AV, Hirst MC et al: Trinucleotide repeat amplification and hypermethylation of a CpG island in FRAXE mental retardation. Cell 1993; 74: 127-143.

4 Knight SJ L, Voelckel MA, Hirst MC, Flannery AV, Moncla A, Davies KA: Triplet repeat expansion at the FRAXE locus and X-linked mild mental handicap. Am J Hum Genet 1994; 55: 81-86.

5 Hamel BCJ, Smits APT, de Graaff E et al: Segregation of FRAXE in a large family: clinical, psychometric, cytogenetic, and molecular data. Am J Hum Genet 1994; 55: 923-931.

6 Mulley JC, Yu S, Loesch DZ et al: FRAXE and mental retardation. Med Genet 1995; 32: 162-169.

7 Carbonell P, Lòpez I, Gabarròn J et al: FRAXE mutation analysis in three Spanish families. Am J Med Genet 1996; 64: 434-440.

8 Gecz J, Gedeon AK, Sutherland GR, Mulley JC: Identification of the gene FMR2, associated with FRAXE mental retardation. Nat Genet 1996; 13: 105-108.

$9 \mathrm{Gu}$ Y, Shen Y, Gibbs RA, Nelson DL: Identification of FMR2, a novel gene associated with the FRAXE CCG repeat and CPG island. Nat Genet 1996; 13: 109-113.

10 Gecz J, Oostra BA, Hockey A et al: FMR2 expression in families with FRAXE mental retardation. Hum Mol Genet 1997; 6: 435-441.

11 Longshore JW, Moore L, Durhan T, Tarleton JC: A novel deletion leading to confusion in the molecular diagnosis of FRAXE (FMR2). Am J Hum Genet Suppl 1996; 59: 2328 (abstract).

12 Zelante L, Gasparini P, Estivill $X$ et al: Connexin26 mutations associated with the most common form of non-syndromic neurosensory autosomal recessive deafness (DFNB1) in Mediterraneans. Hum Mol Genet 1997; 9: 1605-1609.

13 Wechsler D: M easurement of Adult Intelligence. The William Wilkins Company: Baltimore, 1947. 
14 Wechsler D: Wechsler Memory Scale. Psychological Corporation: San Antonio, TX, 1945.

15 Petris L: Visual Retention Test in the examination of visual perception. Organizzazioni Specialia O.S: Florence, 1981.

16 Huber W, Poeck K, Willmes K: Aachen Aphasie Test. Adv Neurol 1984; 42: 291-303.

17 Benton AL, Sivan AB, Hamsher K de S, Varney NR, Spreen O: Judgement of Line Orientation (form H). Iowa City: IO, USA, 1978.

18 Benton AL, Sivan AB, Hamsher K de S, Varney NR, Spreen O: Facial Recognition. Iowa City, IO, USA, 1975.

19 Allingham-Hawkins DJ, Ray PN: FRAXE expansion is not a common etiological factor among developmentally delayed males. Am J Hum Genet 1995; 56: 72-76.

20 Meadows KL, Pettay D, Newman J, Hersey J, Ashley AE, Sherman $S L$ : Survey of the fragile $X$ syndrome and the fragile $X$ E syndrome in a special education needs population. Am J Med Genet 1996; 64: 428-433.

21 Syrrou M, Georgiou I, Grigoriadou M et al: FRAXA and FRAXE prevalence in patients with nonspecific mental retardation in the Hellenic population. Genetic Epidemiol 1998; 15: 103-109.

22 Murray A, Youings S, Dennis N et al: Population screening at the FRAXA and FRAXE loci: molecular analyses of boys with learning difficulties and their mother. Hum Mol Genet 1996; 5: 727-735.

23 Knight SJL, Ritchie RJ, Chakrabarti L et al: A study of FRAXE in mentally retarded individuals referred for fragile $X$ syndrome (FRAXA) testing in the United Kingdom. Am J Hum Genet 1996; 58: $906-913$.
24 Holden JJA, Julien-Inalsingh C, Chalifoux $M$ et al: Trinucleotide repeat expansion in the FRAXE locus is not common among institutionalised individuals with non-specific developmental disabilities. Am J Med Genet 1996; 64: 420-423.

25 Biancalana V, Taine L, Bouix JC et al: Expansion and methylation status at FRAXE can be detected on EcoRI blots used for FRAXA diagnosis: analysis of four FRAXE families with mild mental retardation in males. Am J Hum Genet 1996; 59: 847-854.

26 Brown WT: The FRAXE syndrome: is it time for routine screening? Am J Med Genet 1996; 58: 903-905.

27 Murgia A, Polli R, Vinanzi C et al: Amplification of the Xq28 FRAXE repeats: extreme phenotype variability? Am J Med Genet 1996; 64: 441-444.

28 Milà M, Sànchez A, Badenas $C$ et al: Screening for FM R1 and FMR2 mutations in 222 individuals from Spanish special schools: identification of a case of FRAXE-associated mental retardation. Hum Genet 1997; 100: 503-507.

29 Russo S, Selicorni A, Bedeschi MF et al: Molecular Characterization of FRAXE-positive subjects with mental impairment in two unrelated italian families. Am J Med Genet 1998; 75: 304-308.

30 Reyners E, Vits L, De Boulle K et al: The full mutation in the FMR1 gene of male fragile $X$ patients is absent in their sperm. Nat Genet 1993; 4: 143-146. 